\title{
Billinghurst y las salitreras de Tarapacá (1872-1876) ${ }^{1}$
}

\section{Billinghurst and the Tarapacá saltpeter industry, 1872-1876}

\author{
Carlos Roberto Flores Soria ${ }^{2}$ \\ Universidad Nacional Federico Villarreal. Lima, Perú \\ cflores@unfv.edu.pe
}

\section{RESUMEN}

El artículo tiene como objetivo conocer la visión de Guillermo Billinghurst en su faceta de estudioso del desarrollo de la industria salitrera de Tarapacá en la etapa peruana, especialmente entre 1872 y 1876, años en que gobernó Manuel Pardo y se vio una gran expansión de la producción y exportación del salitre, así como de la intervención del Estado a través del estanco (1873) y la expropiación (1875) de la industria. Billinghurst publicó importantes trabajos que se ocupan de la formación de las empresas salitreras y fue un crítico de la política salitrera del Gobierno civilista.

\section{PALABRAS CLAVE}

Industria salitrera, Tarapacá, Guillermo Billinghurst, estanco, expropiación

\section{ABSTRACT}

The focus of this article is the position of Guillermo Billinghurst in his role as a scholar of the development of the Tarapacá saltpeter industry during its Peruvian phase, particularly from 1872 to 1876, during the government of Manuel Prado, which saw a massive expansion in the production and exporting of saltpeter, and intervention by the state in the form of government licensing (1873) and expropriation (1875) of the industry. Billinghurst published major studies concerned with the development of saltpeter

1 El artículo es una versión reelaborada de la ponencia presentada en el Congreso Nacional de Historia realizado en Trujillo en agosto de 2016.

2 Licenciado en Historia, docente de la Escuela Profesional de Historia, Facultad de Humanidades, Universidad Nacional Federico Villarreal (Lima). 
companies and was a critic of the civilian government's saltpeter policy.

\section{KEYWORDS}

Saltpeter industry, Tarapacá, Guillermo Billinghurst, government licensing, expropriation

\section{Introducción}

Históricamente, Guillermo Billinghurst es un personaje situado en la segunda década del siglo XX, especialmente cuando, entre 1912 y 1914, fue elegido presidente de la República y su mandato terminó con un golpe militar dirigido por Óscar R. Benavides. Su Gobierno llamó la atención por la inspiración de orden «populista» que algunos historiadores le han atribuido y por expresar una temprana ruptura en la llamada Republica Aristocrática. Sin embargo, en las tres últimas décadas del siglo XIX, en la plenitud de su juventud, resulta siendo un personaje desconocido. Los diccionarios históricos-biográficos peruanos solo le dedican unas cuantas líneas.

Jorge Basadre (1983, t. VIII) elaboró una breve biografía destacando los momentos más importantes de la vida de Guillermo Billinghurst: salitrero, escritor y político. El estudioso chileno del mundo salitrero tarapaqueño Sergio González (2000 y 2011) y el sociólogo peruano Osmar Gonzales (2009) coinciden en reconocer que Billinghurst fue un hombre de «frontera» («transfronterizo») y «multifácetico», ya que reunió cualidades de político, empresario, escritor y que sus obras son fundamentales para la historia y geografía regional peruano-chilena del siglo XIX: Tarapacá y su industria salitrera. El reconocido historiador chileno del salitre Óscar Bermúdez (1963) se ocupa de la etapa en que nuestro personaje enfrentaba la política salitrera del Gobierno de Manuel Pardo (1872-1876), contrastando el liberalismo de ambos respecto al estanco y la expropiación.

En 2011, en Chile se publicó Los capitales salitreros de Tarapacá, de Guillermo Billinghurst, acompañado de un notable estudio de Sergio González Miranda en la Biblioteca Fundamentos de la Construcción de Chile.

En estos últimos años la historiografía económica y social peruana, si bien no estudia el papel de Billinghurst en la industria salitrera, ha realizado importantes contribuciones sobre el impacto del salitre en la economía pública, sobre todo los alcances de la política salitrera del Gobierno de Manuel Pardo, expresado en el estanco y la expropiación. Entre ellos, tenemos trabajos de Carmen Mc Evoy (1994, 1997), Carlos Flores (2000), Rory Miller (2011), Miriam Salas (2010, 2011), Javier Tantaleán (1983, 2011), Carlos Contreras (2004, 2012), entre otros. 
El artículo tiene como objetivo conocer la visión de Guillermo Billinghurst acerca del desarrollo de la industria salitrera de Tarapacá de 1872 a 1876. Sus escritos Rápida ojeada sobre la cuestión del salitre (1875), Los capitales salitreros (1889) y Legislación sobre el salitre y bórax en Tarapacá (1903) son importantes para entender cómo se desarrolló la industria salitrera hasta 1879 en el sur peruano, principalmente la formación y composición del capital en la expansión e industrialización del salitre.

\section{Notas sobre el escenario geográfico}

San Lorenzo de Tarapacá fue la antigua capital del Partido de la Intendencia de Arequipa en el Virreinato. En la época republicana pasó a ser provincia y en 1878, departamento. Según Guillermo Billinghurst, en Tarapacá se podían distinguir cinco zonas (Billinghurst, 1893, 1886; Bertrand, 1879): a) la primera se iniciaba en las orillas del mar, continuaba por la serranía de la costa, territorio completamente estéril, que era la zona del guano y de las minas (Huantajaya, Santa Rosa, entre otras); b) la segunda zona se iniciaba en los márgenes de la quebrada de Camarones, limitaba con el desierto de Atacama, terminaba cerca de los márgenes del Loa y era considerada la región salitrera; c) la tercera zona comprendía la pampa del Tamarugal, y limitaba por el norte con la quebrada de Berenguela y por el sur con el río Loa; d) la cuarta zona comenzaba en los márgenes orientales de la pampa del Tamarugal, terminaba en la cordillera y se caracterizaba por la presencia de quebradas, como la de Camarones; e) la quinta zona comprendía la cordillera de los Andes y sus respectivas vertientes. El siguiente mapa ilustra el espacio:

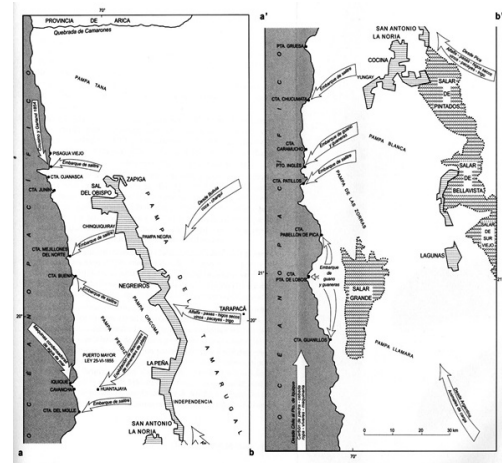

FIGURA 1. Zonas salitreras y puertos en Tarapacá. Fuente: Bermúdez, 1963, pp. 124-125.

Las flechas que se observan en la figura señalan el movimiento del salitre hacia los puertos de embarque. Los puertos que adquirieron notoriedad en la época fueron lquique, considerado el segundo más importante del Perú, seguido por Pisagua y, finalmente, el de Mejillones. En el 
desarrollo de la industria salitrera fue importante la cobertura vegetal que ofrecía la pampa de Tamarugal (Salas, 2010, p. 40). Iquique se convirtió en un puerto que creció y se transformó al ritmo de la exportación del salitre (Donoso 2003, 2007).

\section{Datos biográficos de Guillermo Billinghurst}

Guillermo Eduardo Billinghurst nació en Arica el 27 de julio de 1851. A comienzos del siglo XX, Kaempffer (1914, p. 35), estudioso de la industria del salitre y el yodo chileno, se refería a él como: «El hombre de talento, de ciencia y saber, no solo pertenece a la historia de la nación en la que viera la luz, sino también a aquellas en que su labor es fructífera, llegando así a ser una figura internacional». Su vida transcurrió entre la política ${ }^{3}$, los negocios y acuciosos estudios sobre la región industrial salitrera peruana, hasta antes de la guerra con Chile en 1879. Basadre sostiene que tuvo una juventud borrascosa, como acompañar a Nicolás de Piérola en la llamada Expedición del Talismán de octubre a diciembre de 1874, en el intento de sublevarse contra el Gobierno de Manuel Pardo (Basadre, 1983, tomo VIII, p. 400$)^{4}$.

De 1872 a 1873, a raíz de las primeras medidas del Gobierno civilista sobre el salitre, como el estanco, Billinghurst y un grupo de salitreros se trasladaron desde lquique a Lima para entrevistarse con el presidente Pardo. En ese ínterin, Dávalos y Lissón (1915, pp. 228-229) escribió las primeras semblanzas de la personalidad en 1873:

he amistado con un mozo animado, bromista, ameno, inteligente, nacido en Arica. No tendrá más de 24 años. Es hijo de padre argentino y de abuelo inglés. Se llama Guillermo Billinghurst. Fue secretario de los salitreros tarapaqueños que, bajo la presidencia de don Juan Gildemaister, conferenciaron, en febrero de este año [1873], con don Manuel Pardo.

Según Bermúdez (1963), era un conocedor de la industria salitrera y combatió la política del estanco y de la expropiación implementada por los civilistas, no solo porque observó en ella dificultades en el aspecto

3 De 1878 a 1879 fue diputado por Tarapacá. De 1886 a 1890 se desempeñó como cónsul general del Perú en Chile. Fue miembro del Partido Demócrata y acompañó a Piérola en la toma de Lima en 1895 para derrocar al Gobierno del general Andrés A. Cáceres. En 1895 fue electo primer vicepresidente de la Republica y, a la vez, senador de Moquegua. En 1896 presidió el Senado y el Congreso de la República. En 1898 fue presentado ante el Gobierno chileno como ministro plenipotenciario especial para arreglar la cuestión Tacna y Arica. Negoció el protocolo denominado Billinghurst-Latorre. En 1899 lanzó su candidatura a la presidencia, pero renunció por discrepancias con miembros del pierolismo. Fue alcalde de Lima en 1909 y 1910. En setiembre de 1912 asumió funciones como presidente del Perú y fue derrocado por Óscar R. Benavides en febrero de 1914.

4 También participó en la revuelta del Yacango y en la aventura del Huáscar de 1877. 
pragmático, sino también porque representaba conceptos económicos opuestos a los salitreros. Fue un empresario moderno y un intelectual interesado en el periodismo y en la investigación local y regional. Conocía la dinámica salitrera de Tarapacá, de las compañías, de los capitales, especialmente el inglés. Valparaíso, Tarapacá, Iquique y Lima fueron los ambientes en el que se desenvolvió.

Sergio González (2000 y 2011) y Osmar Gonzales (2009) destacan sus cualidades de investigador e intelectual. Para González (2000, p. 5):

Billinghurst fue un hombre de frontera no solamente por haber nacido en Arica y vivido su adolescencia y madurez en lquique, sino también porque siempre estuvo entre ambos países; por ejemplo, estudió en Valparaíso e hizo su carrera política en Lima. Su pensamiento social y político fue también de frontera; se comprometió en la democratización de su país a través de la participación de las emergentes clases populares.

En esa perspectiva, Osmar Gonzales (2009, p. 41) lo considera como «transfronterizo y multifacético». Para ambos autores, la personalidad y protagonismo de Billinghurst ha sido opacada por la oligarquía y la historiografía peruana ${ }^{5}$. Sin embargo, nuestro personaje fue un atento estudioso de Tarapacá e Iquique. Como veremos, el ciclo vital de su valiosa producción intelectual surge entre 1882 y 1893, en pleno auge económico generado por el nuevo ciclo de la explotación salitrera en lquique, que permitió «el desarrollo cultural donde los clubes sociales, grupos de teatro, logias masónicas, filarmónicas, comenzaron a emerger [...] Entre ellos, se fundó en 1886 el Círculo Literario Ateneo [...] Guillermo Billinghurst [fue] uno de sus fundadores» (González, 2011, p. xvi). Por ejemplo, Estudio sobre la geografía de Tarapacá, publicado en 1886, fue leído en este importante recinto.

Por otro lado, sus preocupaciones sobre la historia del salitre se materializaron en tres importantes libros: Rápida ojeada sobre la cuestión del salitre (1875), Los capitales salitreros (1889) y Legislación sobre el salitre y bórax en Tarapacá (1903). Cada uno refleja la coyuntura y temperamento que Billinghurst le puso, el primero más enérgico y pesimista; el segundo y el tercero, más reflexivos y documentados. Los tres constituyen fuentes de obligada lectura para cualquier estudioso del salitre. Además, sus

5 Para Gonzales (2009), la historiografía peruana no ha sido generosa con Billinghurst. Jorge Basadre, en su Historia de la República (1983, t. VIII), lo considera como un demagogo que pretendió cerrar el Congreso violentando la legalidad establecida. En ninguna lectura de la realidad peruana aparece como personaje importante, a pesar de que con él se iniciaron varios procesos políticos y sociales en el Perú del siglo XX (p. 41). 
preocupaciones sobre agricultura, demografía, ferrocarriles, agua potable, entre otras, se plasmaron en las siguientes publicaciones:

1878 Compendio de la legislación municipal del Perú 1873-77. Iquique. 1880 Alfonso Ugarte, corona fúnebre. Lima.

1880 Reconocimiento militar del río desaguadero y de la altiplanicie andina. Lima.

1886 Estudio sobre la geografía de Tarapacá. Santiago de Chile.

1887 El abastecimiento del agua potable para el puerto de lquique. Lima. 1888 La condición legal de los peruanos nacidos en Tarapacá. Santiago de Chile.

1889 Documentos relativos a la salitrera Lagunas. Santiago de Chile.

1893 La irrigación de Tarapacá. Santiago de Chile.

1905 Documentos relativos al ferrocarril de Patillos. Valparaíso.

1915 El presidente Billinghurst y la nación. Santiago de Chile.

\section{Billinghurst y el capital salitrero}

El desarrollo de la industria salitrera solo puede ser entendido a partir de tres elementos: capital, tecnología y mercados. A diferencia del guano que se extraía directamente de las islas con mínima inversión, el salitre involucraba diversos procesos que iban desde su extracción hasta los mercados europeos. Después de la ocupación chilena de Tarapacá, en noviembre de 1879, y la culminación de la guerra, en 1884, se publicaron importantes estudios que daban cuenta de la historia del salitre en la «era peruana» y del inicio del ciclo salitrero en la economía chilena. Entre ellos tenemos el de Alejandro Bertrand (1892), de Semper y Michels (1908) y de Enrique Kaempffer (1914).

Los dos primeros se ocupan del desarrollo de la industria salitrera a partir de la ocupación de los terrenos, las propiedades físico-químicas del caliche, la formación del capital y la propiedad, del trabajo y de las relaciones laborales, de la capacidad productiva y de los costos, de los ferrocarriles, de los costos de embarque y exportación a mercados europeos, y, por último, del estanco y la expropiación en la «era peruana», importante información documental de carácter legal acompaña sus anexos. Kaempffer se ocupa de los hombres vinculados a la industria salitrera, los antecedentes históricos, los cateos, las técnicas, la extracción, la elaboración del salitre y la importancia del agua en todo el proceso.

Los aportes de Billinghurst acerca de la industria salitrera sirvieron de base para sustentar y organizar ese cuerpo de conocimiento que exponen los autores antes citados. 
Entonces, ¿cómo y cuándo se inicia la historia industrial del salitre? Según Billinghurst, la elaboración y exportación del salitre (Le y Dagnino, 1893 , p. 5) ${ }^{6}$, cuya materia era el caliche ${ }^{7}$ de la provincia de Tarapacá, no tiene su origen en 1830. Desde los puertos de lquique y Pisagua se exportó, muchos años antes de esa fecha, salitre para España. Mientras Lima y algunos espacios regionales se involucraban en las luchas por la independencia, en Tarapacá la emergente industria salitrera iba adquiriendo notoriedad. En ese proceso, Billinghurst destaca el aporte de Tadeo Haenke, investigador naturalista que llegó al Perú en 1790 en la misión científica dirigida por Alejandro Malaspina, en el procedimiento de «separar y beneficiar el caliche»; así como de Sebastian Ugarrisa y Matías de la Fuente, personajes que alcanzaron notoriedad debido a sus esfuerzos dedicados a construir oficinas salitreras y realizar embarques de salitre hacia Lima (Billinghurst, 1889a, p. 11) ${ }^{8}$.

De 1810 a 1812 se construyeron, en las pampas salitreras de Negreiros, Pampa Negra y Zapiga, entre siete $u$ ocho oficinas ${ }^{9}$ para elaborar salitre. Para tal propósito se adoptaron los antiguos fondos de beneficiar la plata. Ugarrisa buscó convertir la explotación del salitre en una industria atractiva para los negocios y fue seguidos por el científico Mariano Eduardo de Rivero, quien en 1820 dio a conocer los beneficios del nitrato de soda de Tarapacá en Europa. Al respecto, en 1821 escribió:

Hasta ahora ninguna obra de minerología había hablado del nitrato de soda nativo, ya que acaba de descubrirse esta sal, y en bastante cantidad, en el distrito del Perú llamado Tarapacá. Forma en él una capa de varios pies de grueso, mostrándose, en algunos lugares, a la superficie, y ocupando una extensión de más de 40 leguas.

Se encuentra el nitrato de sosa cerca de lquique, puerto del sur del Perú, en el cual hay mas de 60000 quintales de esta sal purificados por

6 El nitrato de soda es conocido también con los nombres de salitre sódico, salitre cúbico, nitrato sódico, salitre de Tarapacá y salitre de Chile (Le y Dagnino, 1893, p. 5).

7 Conglomerados de detritus unidos por un cemento de sales y que contienen la mayor cantidad de nitrato en relación con las capas anteriores (Bermúdez, 1963, p. 408). Para Echevarría (1934, p. 10), el caliche es una «mezcla de sales y sustancias insolubles en agua, en la que predomina el nitrato de sodio mezclado con cloruros y sulfatos, de la que se extrae el salitre; se presenta cementada en mantos o capas horizontales».

8 Billinghurst (1889a, p. 11) anota: «Por aquellos años, de octubre de 1812 a febrero de 1813, la producción llegó a la cantidad de 23160 quintales [y] 31 libras. Para esa misma fecha, Ugarrisa envió al Callao siete cargamentos de nitrato de soda por un total de 22 723,49 quintales, y el precio pagado fue de $\$ 35585,25$ y el flete de las oficinas a la costa fue de $\$ 10329,50 »$.

9 Según Bermúdez (1963, p. 416), la palabra oficina se encuentra en los documentos administrativos de los asientos mineros de entonces. Parece que la asociación de oficina y salitrería determinó el nombre de oficina salitrera que terminó por reemplazar al de minas de salitre. 
disolución y cristalización y podrán abastecerse los traficantes europeos (Ugarrisa, 1857, tomo l, pp. 5-6).

Rivero auguró el futuro de esta industria al señalar las grandes ventajas de este descubrimiento. No se equivocó. Aproximadamente hasta 1850, según Billinghurst, los industriales que se habían conectado con la explotación y producción del salitre de Tarapacá «eran todos peruanos, de naturales de Tarapacá, con excepción de don Jorge Smith, que se había establecido en los salitrales de la Nueva Noria» (Billinghurst, 1889a, p. 13).

Jorge Smith fue un personaje salitrero muy importante para la época. En compañía de William Bollaert, por encargo de Ramón Castilla, entonces intendente de la provincia de Tarapacá, exploró en 1827 la llamada pampa de Tamarugal. Boellert fue un estudioso de la geografía de Tarapacá, natural de Inglaterra, y llegó al Perú en 1825 (Bollaert, 1975; Castro, Guerrero, Silva y Silva, 2017, pp. 121-164). Entre 1850 y 1870 se dedicó a publicar sus trabajos acerca de las descripciones y expediciones realizadas (Bermúdez, 1975, pp. 314-318), y con el español José Sandes fueron los primeros extranjeros que se dedicaron en Tarapacá a la industria salitrera. En 1852 se asocian y forman la sociedad industrial que más tarde se llamó Compañía de Salitres de Tarapacá (Kaempffer, 1914, p. 119). Según Kaempffer, «el señor Jorge Smith fue un esforzado industrial; a su iniciativa se deben muchos adelantos en la elaboración del salitre y en la viabilidad de Tarapacá (Kaempffer, 1914, p. 119) ${ }^{10}$.

Para Billinghurst, 1853 es un año que inicia una nueva época en la historia de la industria salitrera. El industrial chileno Pedro Gamboni, después de varios experimentos realizados en Estados Unidos y en sus oficinas salitreras de Tarapacá, logró aplicar el vapor a la disolución del caliche, en reemplazo del antiguo método de fuego directo que era costoso (Kaempffer, 1914, p. 179). Para el 2 de noviembre de 1853, el Gobierno peruano le concedió el privilegio exclusivo para usar el sistema de elaboración por cinco años (Billinghurst, 1889a, p. 14).

En 1856, Jorge Smith construyó una máquina ${ }^{11}$ en la Nueva Noria, la que, al cabo de algunos años y tras experimentar transformaciones, se convirtió en la conocida maquina Limeña, que tenía una capacidad de producción de 200000 quintales anuales (Billinghurst, 1889a, p. 14; Kaempffer, 1914, p. 119). Así, años después se construyeron las siguientes:

10 Kaempffer, también, destaca a Juan Williamson, natural de Escocia, por su contribución al desarrollo de la industria del salitre, al instalar a gran escala explotaciones salitreras en la apartada región sur de Tarapacá. En 1852 era propietario de 10 oficinas de paradas que después darían lugar a la máquina Alianza.

11 Máquina, según Echevarría (1934), «es el establecimiento de elaboración del caliche, en su conjunto» (p. 23). 
TABLA 1. Máquinas salitreras construidas de 1856 a 1862

\begin{tabular}{|c|c|c|}
\hline Máquina & Propietario & Lugar \\
\hline Huanza & Fernando Corssen & Cocina \\
\hline Salar & Federico Freraut & La Noria \\
\hline La China & Demetrio Figueroa & La Noria \\
\hline Victoria & Soruco y Ca. & En el norte \\
\hline Carolina & Jorge Smith y Ca. & En el norte \\
\hline
\end{tabular}

Fuente: Elaboración propia, basada en datos de Billinghurst, 1889a, p. 14.

La primera máquina de elaborar salitre fue construida en una de las oficinas de Sal de Obispo. La segunda, edificada personalmente por Gamboni, en «Sebastopol» y la tercera en "Cocina», cuyos terrenos eran de propiedad de la Oficina «San Pedro» de Gildemeister y Ca. (Billinghurst, 1889a, p. 14) ${ }^{12}$. Billinghurst señala tres momentos:

a. 1870 y 1872: la presencia de máquinas en Tarapacá se había incrementado y la estimación de la producción iba alcanzando proporciones no vista antes, tal como apreciamos en la siguiente tabla:

TABLA 2. Máquinas, capacidad producción y propietarios (1870-1872)

\begin{tabular}{|c|c|c|c|}
\hline Establecimiento & Capacidad de producción anual en quintales & $\%$ & Propietario \\
\hline Carolina & 300000 & 9,3 & Compañía de Salitres de Tarapacá \\
\hline Argentina & 300000 & 9,3 & J. Gildemeister y Ca. \\
\hline Solferino & 280000 & 8,7 & Félix Massardo \\
\hline San Pedro & 250000 & 7,8 & J. Gildemeister y Ca. \\
\hline Victoria & 220000 & 6,9 & Soruco y Ca. \\
\hline San Antonio & 200000 & 6,3 & Hainsworth y Ca. \\
\hline Porvenir & 200000 & 6,3 & La Fuente y Sobrino \\
\hline Peruana & 200000 & 6,3 & Virginia Loayza \\
\hline Sacramento & 200000 & 6,3 & José Manuel de Loayza \\
\hline La Nueva Noria & 200000 & 6,3 & Compañía de Salitres de Tarapacá \\
\hline Palacio Industrial & 120000 & 3,7 & Nugeat y Caplong \\
\hline Germania & 120000 & 3,7 & Juan Vernal y Castro \\
\hline San Carlos & 120000 & 3,7 & Eugenio Marquezado \\
\hline Santa Isabel & 120000 & 3,7 & Pedro Elquera \\
\hline
\end{tabular}

12 Juan Gildemeister nació en 1823. Era natural de Bremen (Alemania) y a mediados del siglo residía en Lima. Se interesó por el salitre a comienzos de 1860 y se convirtió en habilitador de salitreros. Después del terremoto de 1868 se dedicó a la producción del nitrato. De 1870 a 1874 adquirió las oficinas Huanza, Argentina, San Pedro y San Juan (Centro de Estudios Miguel Enríquez, s/f, p. 3). 


\begin{tabular}{|c|c|c|c|}
\hline Santa Rita & 110000 & 3,4 & José María González Vélez \\
\hline La China & 100000 & 3,1 & Demetrio Figueroa \\
\hline Huanza & 100000 & 3,1 & J. Gildemeister y Ca. \\
\hline Rosario & 60000 & 1,9 & Manuel M. Pérez \\
\hline Total & 3200000 & 100 & \\
\hline
\end{tabular}

Fuente: Billinghurst, 1889, p. 15.

$\%=$ porcentaje de participación

b. 1872-1874: se construyeron nuevas máquinas y la capacidad de producción casi triplicaba a la fase anterior. Veamos la siguiente tabla:

TABLA 3. Nuevas máquinas, capacidad producción y propietarios (1872-1874)

\begin{tabular}{|c|c|c|c|}
\hline Establecimiento & Capacidad de producción anual en quintales & $\%$ & Propietario \\
\hline Limeña & 600000 & 6,88 & Compañía de Salitres de Tarapacá \\
\hline Nueva Soledad & 460000 & 5,27 & Juan Cauvi \\
\hline Peña Grande & 450000 & 5,16 & Compañía Salitrera de la Peña \\
\hline Valparaíso & 450000 & 5,16 & Compañía Salitrera Valparaíso \\
\hline Candelaria & 450000 & 5,16 & Benavidez \\
\hline Santa Catalina & 400000 & 4,58 & Compañía Salitrera Americana \\
\hline Lagunas & 360000 & 4,13 & Compañía Salitrera Esperanza \\
\hline La Perla & 360000 & 4,13 & Compañía Salitrera Rímac \\
\hline Santa Laura (w) & 300000 & 3,44 & Guillermo Wendell \\
\hline Palma & 300000 & 3,44 & Peruvian Nitrate Company \\
\hline Alianza & 300000 & 3,44 & Compañía de Salitres Alianza \\
\hline San Lorenzo & 300000 & 3,44 & Ugarte, Cevallos y Ca. \\
\hline Esmeralda & 300000 & 3,44 & Clark, Erick y Ca. \\
\hline San Vicente & 300000 & 3,44 & Granadino y Hnos. \\
\hline Máquina Negreiros & 300000 & 3,44 & Compañía Salitrera Negreiros \\
\hline Resurrección & 300000 & 3,44 & Pedro J. Zavala \\
\hline Paposo & 240000 & 2,75 & Fölsen y Martín \\
\hline Matillana & 240000 & 2,75 & Morales y Ca. \\
\hline Providencia & 240000 & 2,75 & Compañía Salitrera Providencia \\
\hline Nueva Carolina & 220000 & 2,52 & Compañía Salitrera Nueva Carolina \\
\hline Peña Chica & 210000 & 2,40 & Aguirre y Oviedo \\
\hline Trinidad & 210000 & 2,40 & J. Carpio y Ca. \\
\hline San Agustín & 200000 & 2,29 & Juan Francisco Balta \\
\hline San José (Noria) & 180000 & 2,06 & Devéscovi y Arredondo \\
\hline San Andrés & 180000 & 2,06 & M. María Pérez \\
\hline Carmen Bajo & 180000 & 2,06 & Compañía Salitrera Rímac \\
\hline
\end{tabular}




\begin{tabular}{|c|c|c|c|}
\hline Carmen Alto & 150000 & 1,72 & Orriols y Ca. \\
\hline Bearnés & 140000 & 1,60 & Francisco Layous \\
\hline Cala Cala & 120000 & 1,37 & Eufemía C. de Hidalgo \\
\hline Santa Laura (Borra) & 100000 & 1,14 & Barra y Riesco \\
\hline Pozo de Almonte & 80000 & 0,91 & Fernando López \\
\hline El Morro & 50000 & 0,57 & Manuel E. Riveros \\
\hline Tarapaqueña & 45000 & 0,51 & Matías Vicentelo y Ca. \\
\hline Total & 8715000 & 100 & \\
\hline
\end{tabular}

Fuente: Billinghurst, 1889a, p. 16

$\%=$ porcentaje de participación

c. 1874 y 1878: continuó la expansión de la industria salitrera, pero en menor proporción. Se incorporaron nuevas máquinas, como:

TABLA 4. Nuevas máquinas, capacidad de producción y propietarios (1874-1878)

\begin{tabular}{|c|c|c|c|}
\hline Establecimiento & Capacidad de producción anual en quintales & $\%$ & Propietario \\
\hline Agua Santa & 500000 & 10.03 & J.D. Campbell \\
\hline California & 360000 & 7.22 & Compañía de Salitrera California \\
\hline San Juan & 360000 & 7.22 & J. Gildemeister y Ca. \\
\hline Emp. Barrenechea & 360000 & 7.22 & Compañía Salitrera Barrenechea \\
\hline Ángeles & 300000 & 6.02 & Mariano Mendizabal \\
\hline Santa Ana & 300000 & 6.02 & Pflucker y Ca. \\
\hline San Pablo & 300000 & 6.02 & Pedro Elguera \\
\hline San Francisco & 300000 & 6.02 & Evaristo Brañes \\
\hline Santa Adela & 210000 & 4.21 & José Devéscovi \\
\hline Dolores & 210000 & 4.21 & Juan Cobo \\
\hline Católica & 210000 & 4.21 & Juan Bacigalupo \\
\hline Magdalena & 200000 & 4.01 & J. Manuel Montero \\
\hline Chinquiquiray & 200000 & 4.01 & Oviedo y Trillo \\
\hline Camiña & 200000 & 4.01 & Mariano Ossio \\
\hline San Antonio (P) & 200000 & 4.01 & Eusebio Peñaranda \\
\hline Angela & 200000 & 4.01 & Loayza y Pascal \\
\hline Carmelita & 150000 & 3.01 & Domingo Lecaros. \\
\hline Concepción & 120000 & 2.40 & Compañía de Salitrera California \\
\hline Salar de Noria & 110000 & 2.20 & Ugarte, Cevallos y Ca. \\
\hline San Juan (Soledad) & 100000 & 2.0 & José Romero \\
\hline San José (Yungay) & 48000 & 0.96 & Manuel E. Riveros \\
\hline Buen Retiro & 45000 & 0.90 & Quiroga y Hermano \\
\hline Total & 4983000 & 100 & \\
\hline
\end{tabular}

Fuente: Billinghurst, 1889a, p. 17.

$\%=$ porcentaje de participación 
Como se puede apreciar, hasta aproximadamente 1865, la industria salitrera se desarrolló a base de esfuerzos individuales. Pero a partir de los años siguientes el surgimiento de nuevos centros de producción fue vertiginoso y, consecuentemente, se adquirió una nueva modalidad de impulsar la inversión en la industria salitrera: el crédito bancario. Esta modalidad iría desplazando al sistema de habilitaciones dominante hasta entonces.

Como sugiere Billinghurst, la capacidad de producción de las máquinas fue en ascenso, teniendo en cuenta los tres periodos antes mencionados (Billinghurst, 1889a, p. 18):

TABLA 5. Capacidad de producción

\begin{tabular}{ccc} 
Años & Unidad de peso & al año \\
\hline 1870 a 1872 & quintales & 3200000 \\
\hline 1872 a 1874 & quintales & 11715000 \\
\hline 1874 a 1878 & quintales & 16363000 \\
\hline
\end{tabular}

Fuente: Elaboración propia, basada en datos de Billinghurst, 1889a, p. 18.

La producción fue en ascenso. Factores externos explican ese crecimiento. Luis Benjamín Cisneros sostiene que las causas que favorecieran el aumento de la demanda y exportación del salitre:

se explica por la disminución de las ventas del guano desde 1869 y el alza del salitre especialmente entre 1870 y 1871 , porque tanto como la guerra franco-prusiana dañó la agricultura en Francia, Alemania y Bélgica, favoreció la confección de la pólvora por medio del nitrato de soda, uno de sus principales agentes vigorosos ${ }^{13}$.

Por otro lado, de 1870 a 1876 el precio fue variando entre 15 y 12 chelines el quintal (Hernández, 1930, p. 90).

Conviene precisar que fueron tres los sistemas de elaboración y producción del salitre que se conocieron: paradas (1810-...), máquinas (18531885) y shanks (1876-1942) (Crozier, 1997, pp. 56-126). En este proceso, es importante destacar que la producción en las llamadas paradas ${ }^{14}$ como $^{2}$

13 Citado por Antonio Raimondi en 1875, p. 6. Luis Benjamín Cisneros fue un destacado intelectual, poeta y político de la época, vinculado a los civilistas.

14 La denominación de parada se difundió en Tarapacá de 1810 a 1830. Parada y paradero designan un lugar en que se hace estación en un viaje y puesto que allí se para. Los primitivos lugares en que se explotó el salitre, como Zapiga y Pampa Negra, eran paraderos en el escaso tráfico que hacía entonces por la pampa Tamagural. Pero el nombre de paradas puede estar asociada asociado también a la técnica elaborada. Los interesados en explotar salitre iban a la pampa a parar sus fondos para elaborar. Los fondos eran puestos de a pares, uno a cada lado de la hornilla. Aplicado el nombre primeramente solo al conjunto de aparatos con que se elaboraba, luego se hizo extensivo a toda la explotación y se decía «las aradas» en el sentido de «oficinas salitreras» (Bermúdez, 1963, p. 416). Según el Vocabulario salitrero (1934), de Aníbal Echevarría y Reyes, paradas «es un conjunto de fondos o calderos 
sostiene Billinghurst (Billinghurst, 1889a, p. 18), estuvo predominantemente en manos de industriales peruanos que trabajaban con capital propio. La nacionalidad del capital de 1870 a 1872 se puede apreciar en la siguiente tabla:

TABLA 6. Nacionalidad de capitales en las salitreras (1870-1872)

\begin{tabular}{cl} 
Nacionalidad & Producción anual \\
\hline Capital peruano & 930000 quintales \\
\hline Capital chileno & 800000 quintales \\
\hline Capital inglés & 700000 quintales \\
\hline Capital alemán & 650000 quintales \\
\hline Capital francés & 120000 quintales \\
\hline
\end{tabular}

Fuente: Billinghurst, 1889a, p. 19.

En lo que respecta la última fase de la industria salitrera bajo la administración peruana, es decir, de 1874 a 1878 (Billinghurst, 1889a, p. 19), la tendencia no cambió y se presentó de la siguiente manera:

TABLA 7. Nacionalidad de capitales en las salitreras (1874-1878)

\begin{tabular}{|c|c|c|}
\hline Nacionalidad & Producción anual & Porcentaje \\
\hline Capital peruano & 9583000 quintales & 58,5 \\
\hline Capital chileno & 3120000 quintales & 19,0 \\
\hline Capital inglés & 2200000 quintales & 13,5 \\
\hline Capital alemán & 1250000 quintales & 8,0 \\
\hline \multirow[t]{2}{*}{ Capital italiano } & 210000 quintales & 1,0 \\
\hline & 16363000 & 100,00 \\
\hline
\end{tabular}

Fuente: Billinghurst, 1889a, p. 19, y Basadre, 1983, p. 305.

$\%=$ porcentaje de participación

En tal sentido, la presencia del capital peruano en la industria salitrera fue dominante, si se considera la producción anual. Por ello, Billinghurst sostenía que:

no han sido los capitales ingleses, sino los capitales peruanos primero y los capitales chilenos después, los que han dado vida a la industria del nitrato de soda; y los que, no sin fuertes y frecuentes quebrantos, la han colocado en el brillante pie en que ahora la contemplan los capitales ingleses» (Billinghurst, 1889a, p. 9).

de fierro, que se colocaban sobre hornillos, en los que, primitivamente, se lixiviaba el caliche, por medio de fuego directo» (p. 25). 
De acuerdo con las tablas presentadas, en el desarrollo de la industria salitrera, el capital peruano invirtió más del $50 \%$ en los establecimientos salitreros, respecto al $19 \%$ del chileno y del $13,5 \%$ de procedencia inglesa. En este proceso, según las estimaciones de Billinghurst, las propiedades inglesas no habrían alcanzado ni el $8 \%$ de aquella suma. Pues los capitales ingleses comercialmente no eran tales. Por ejemplo, la Compañía de Salitres de Tarapacá se formó en Tacna mediante escritura pública, el 8 de noviembre de 1865. El capital de esta sociedad fue de 450000 soles, dividido en 12 acciones de 37500 soles cada uno (Billinghurst, 1889a, p. 34). Esta nueva sociedad estuvo integrada por los siguientes accionistas:

El capital de esta sociedad fue de 450,000 soles, dividido en 12 acciones de 37500 soles cada uno. Esta nueva sociedad estuvo integrada por los siguientes accionistas: Guillermo Gibbs con 7 acciones de S. 262 500, Jorge Smith con 3 acciones de 112500 y Melbourne Clark con 2 acciones de 75000 (Billinghurst, 1889a, p. 35).

Según Billinghurst, esta sociedad se formó exclusivamente para liquidar a la anterior empresa, denominada Jorge Smith y Ca., que años atrás se había endeudado con la Casa Gibbs de Valparaíso por una habilitación (préstamo) para la construcción de la maquina Carolina. Así, el aporte de Gibbs y $C a$. representaba el único capital inglés que participaba en esa sociedad. A fines de 1871 , luego de una compleja operación financiera realizada en Londres, la Casa Gibbs participó activamente en los negocios de Smith y en la industria salitrera (Billinghurst, 1889a, p. 32) ${ }^{15}$. Para el año en que Gibbs compró las acciones, los negocios del salitre habían generado grandes expectativas para cualquier industrial deseoso de hacer inversiones (Ravest, 2008, pp. 63-77).

Otro ejemplo de cómo la industria salitrera se formó con capitales de diversas nacionalidades, fue el de J. D. Campbell y compañía, propietarios de las oficinas San Antonio, Agua Santa y Encañada. Una parte era de procedencia inglesa y otra de familias peruanas y bolivianas (Billinghurst, 1889a, p. 33).

El capital que sirvió para financiar la construcción de la máquina Esmeralda fue de origen, en su mayor parte, de industriales ingleses, pero tuvo su origen en Tarapacá. La oficina La Palma, de propiedad de Manuel Almonte y Vigueras, fue comprada por una sociedad inglesa constituida

15 Según Billinghurst, las acciones de Smith y de Clark estaban representadas por los terrenos salitrales de ambos industriales, que representaban un aporte del capital netamente nacional. La participación de Smith en esta sociedad se extendió hasta el 6 de diciembre de 1871 , fecha en que sus testadores traspasaron en Londres los derechos de las tres acciones a la Casa Gibbs; igualmente, Clark traspasó sus dos acciones el 31 de octubre de 1872 a la citada casa, valorizada en 28 233,19,5 libras esterlinas. 
en Londres que se llamó The Peruvian Nitrate Company. El capital con que se formó esta nueva sociedad fue de 250000 soles, y, de acuerdo con Billinghurst, algunos comerciantes de Arequipa fueron sus accionistas (Billinghurst, 1889a, p. 33).

Lima también fue el escenario de la formación de algunas compañías interesadas en participar de la industria salitrera de Tarapacá. De 1870 a 1873, años del despegue de la gran demanda de salitre, se formaron en Lima varias sociedades que contribuyeron en el crecimiento de la producción salitrera. La Compañía Salitrera Barrenechea se constituyó en noviembre de 1871 con un capital de 1000000 de soles, con el propósito de explotar los terrenos salitrales de La Unión y Argentina. Se aplicó para ello una tecnología denominada «método de solución en frío y transporte de los caldos por cantería hasta el puerto» (Billinghurst, 1889a, p. 34).

La Compañía Salitrera Esperanza se formó el 14 de setiembre de 1870. El capital originario fue de 50000 soles y se incrementó en diciembre de 1872 a 210000 soles. También el Banco de Lima le prestó la cantidad de 1000000 soles. Buscaba explotar 408 estacas de terreno salitral ubicadas en el punto conocido como Lagunas (Billinghurst, 1889b) ${ }^{16}$.

Para diciembre de 1871 se formaba la sociedad Ilamada Alianza, cuyo capital pasó de 200000 soles a 320000 soles. Esta compañía tuvo el propósito de comprar las propiedades salitreras de Ángel Custodio Gallo, quien desde 1852 las poseía en Cantón del Sur. Estos terrenos estaban compuestos por 204 estacas, situadas unas en Bellavista, algunas en el sur viejo y otras en Challacollito. El 11 de enero de 1872, por la suma de \$200 000 (pesos chilenos), cantidad pagada por el Banco de Chile, Gallo transfirió sus derechos a la sociedad Alianza (Billinghurst, 1889a, p. 34).

Años después se formaron otras sociedades: La Peña, La Providencia y Rímac. La siguiente tabla resume la formación de las sociedades mencionadas:

TABLA 8. Capital peruano de las compañías salitreras

\begin{tabular}{ccc} 
Nombre & Capital total & $\%$ \\
\hline Compañía Salitrera Esperanza & 1260000 & 34,8 \\
\hline Compañía Salitrera Barrenechea & 1000000 & 27,6 \\
\hline Compañía Salitrera La Peña & 440000 & 12,1 \\
\hline Compañía Salitrera Alianza & 320000 & 8,8 \\
\hline
\end{tabular}

16 Al respecto, Billinghurst estudió la formación de esta compañía. 


\begin{tabular}{ccc}
\hline Compañía Salitrera Rímac & 300000 & 8,3 \\
\hline S/.3620000 & 100 \\
\hline
\end{tabular}

Fuente: Billinghurst, 1889a, p. 35

$\%=$ porcentaje de participación

Antes de la implementación del estanco (1873) y de la expropiación (1875), los bancos de la capital no fueron ajenos a esta industria. El primero en establecerse fue el Banco de Londres, Méjico y Sud América, que ya tenía una sucursal en lquique, con el propósito de facilitar créditos a favor de los industriales salitreros. El Banco Nacional del Perú, fundado el 31 de agosto de 1872, con un capital de 12000000 de soles, inició sus actividades el 10 de setiembre (Camprubi, 1957, p. 4) ${ }^{17}$. A fines de ese año, estableció en lquique una sucursal y otorgó créditos a los industriales. Según Billinghurst, «las cuentas de habilitación de los salitreros alcanzaron hasta la suma de 4000000 de soles» (Billinghurst, 1889a, p. 35). Su aporte a la prosperidad de la industria salitrera de 1872 a 1874, a decir de nuestro autor, fue importante. Un diario de lquique, El Heraldo Americano, refiere Camprubi (1957), informaba que los bancos allí existentes (Banco de Londres, Méjico y Sud América y Banco de Nacional del Perú) «están prestando provechosas facilidades a las transacciones de comercio. Inspiran ilimitada confianza y no se dude que reportan buenas facilidades» (citado por Camprubi, 1957, p. 106).

Un balance sobre la participación del capital «limeño» (Banco Nacional y capitalistas) en la industria salitrera podría considerarse como dominante en la medida en que este aportó aproximadamente 8000000 de soles, esto es, cerca de un millón y medio de libras esterlinas (Billinghurst, 1889a, p. 36).

Por otro lado, la participación del capital chileno en la industria salitrera de Tarapacá también fue notoria. De 1854 a 1864 varias casas comerciales chilenas de Valparaíso facilitaron créditos a los salitreros, que, en su mayoría, no fueron cubiertos por la fuerte baja que experimentó el salitre. Pero el auge del crédito ocurrió de 1870 y 1873, años en que los salitreros de Iquique y Pisagua contrajeron importantes deudas en el mercado de Valparaíso (Pinto, 1987, pp. 119-134). La Compañía Chilena de Consignaciones invirtió capitales en habilitaciones a las salitreras por aproximadamente 1500000 de pesos (Billinghurst, 1889a, p. 37). El Banco Edwards comprometió más de 1000000 de pesos, al realizar algunos adelantos

17 El autor menciona que la fundación de este banco estimuló la competencia y generó que tanto el Banco del Perú como el de Lima incrementaran sus capitales, de 8 y 5 millones, respectivamente. 
a cierto número de salitreros de lquique. Entonces, las relaciones entre Tarapacá y Valparaíso no se limitaron a esos montos. Buscaron tener presencia financiera simultáneamente cuando se desataba vertiginosamente el «furor salitrero» en Londres, que dio paso a la formación de diversas sociedades interesadas en participar en el norte y centro de Tarapacá. El siguiente cuadro ofrece una relación de esas sociedades y señala el capital que invirtieron:

TABLA 9. Capitales chilenos en las salitreras (1870-1876)

\begin{tabular}{|c|c|c|}
\hline Nombre & Capital en \$ & $\%$ \\
\hline Compañía Salitrera Peruana & 520000 & 12,3 \\
\hline Compañía Salitrera Sacramento & 500000 & 11,8 \\
\hline Compañía Salitrera Solferino & 450000 & 10,7 \\
\hline Compañía Salitrera Pisagua & 400000 & 9,5 \\
\hline Compañía Salitrera América & 400000 & 9,5 \\
\hline Compañía Salitrera Nueva Carolina & 400000 & 9,5 \\
\hline Compañía Salitrera Chucumata & 400000 & 9,5 \\
\hline Compañía Salitrera San Carlos & 350000 & 8,3 \\
\hline Compañía Salitrera Negreiros & 300000 & 7,1 \\
\hline Compañía Salitrera Valparaíso & 300000 & 7,1 \\
\hline \multirow[t]{2}{*}{ Compañía Salitrera California } & 200000 & 4,7 \\
\hline & $\$ 4200000$ & 100 \\
\hline
\end{tabular}

Fuente: Billinghurst, 1889a, pp. 37-38.

$\%=$ porcentaje de participación $\quad \$=$ pesos chilenos

De esta manera, Billinghurst destaca que los capitales chilenos radicados en Valparaíso también contribuyeron en impulsar el desarrollo en la industria salitrera desde 1870, con más de 6200000 de pesos.

\section{A modo de conclusión}

Los escritos de Guillermo Billinghurst resultan fundamentales para comprender la formación de la industrial del salitre en Tarapacá durante la Ilamada Era Peruana. La elaboración del salitre requirió de capital, tecnología, mercados, fuerza de trabajo y, sobre todo, de una materia prima que se encontraba en un medio geográfico árido y rico. Contradicciones de la naturaleza, escribió la historiadora Miriam Salas. Billinghurst dio cuenta de la participación de los capitales peruanos, chilenos e ingleses y de otras nacionalidades en el despegue de la industria salitrera. Con ello, ofreció una arista de esa historia que se desarrolló en el sur del país. Sin embargo, hay muchas historias que se entretejieron al compás de la importancia que fue adquiriendo el salitre en Tarapacá e lquique. 
En estos últimos años, la historiografía peruana y chilena viene realizando notables contribuciones acerca de la historia de la industria salitrera en la Era Peruana. Ahora podemos entender cómo el sur peruano no fue ajeno a las políticas de los Gobiernos de turno que buscaron dentro del modelo económico tributario asegurar los recursos naturales para generar y captar una renta a favor de la precaria finanza del Estado, sin que colisionara con los intereses de la élite económica y política comprometida, primero en los negocios del guano y luego en los del salitre. La agenda todavía sigue pendiente. 


\section{REFERENCIAS BIBLIOGRÁFICAS}

Basadre, J. (1983) Historia de la República del Perú. Lima: Editorial Universitaria.

Bermúdez, O. (1968) Historia del salitre desde sus orígenes hasta la guerra del Pacífico. Santiago de Chile: Ediciones de la Universidad de Chile.

(marzo-diciembre de 1975). Esbozo biográfico de WiIliam Bollert. Revista de Geografía Norte Grande, vol. I, nro. 3-4, pp. 314-318.

Bertrand, A. (1892). Condición actual de la propiedad salitrera en Chile. Santiago de Chile: Imprenta Nacional.

(1879). Departamento de Tarapacá. Aspecto general del terreno, su clima i sus producciones. Santiago de Chile: Imprenta de la República.

Billinghurst, G. (1875). Rápida ojeada sobre la cuestión salitre. Valparaíso: Imprenta del Mercurio.

(1886). Estudio sobre la geografía de Tarapacá. Santiago de Chile: Imprenta de El Progreso.

(1889a). Los capitales salitreros de Tarapacá. Santiago de Chile: Imprenta de El Progreso.

(1889b). Documentos relativos a la salitrera Lagunas. Recopilados por Guillermo Billinghurst. Santiago de Chile: Imprenta de El Progreso.

Imprenta y Librería Ercilla.

(1893). La irrigación de Tarapacá. Santiago de Chile:

(1903). Legislación sobre el salitre y bórax en Tarapacá.

Santiago de Chile: Imprenta Cervantes.

Bollaert, W. (1975 [1860]). Descripción de la provincia de Tarapacá. Norte Grande, vol. I, nros. 3-4, pp. 459-479.

Camprubi, C. (1957). Historia de los bancos en el Perú (1860-1879). Lima: Lumen.

Castro, L.; Figueroa, C.; Guerrero, P. y Silva, B. (julio-diciembre de 2017). William Bollaert y sus descripciones geográficas, cartográficas y antropológicas sobre la provincia de Tarapacá en la etapa inicial de la formación republicana del Perú, 1827-1854. HistoReLO, vol. 9, nro. 18, pp. 121-164.

Centro de Estudios Miguel Enríquez (2007). La industria salitrera de Tarapacá. Recuperado de http://www.archivochile.com/Historia_de_Chile/ sta-ma2/2/stamatexrel000008.pdf 
Contreras, C. (2004). El aprendizaje del capitalismo. Estudios de historia económica y social del Perú republicano. Lima: Instituto de Estudios Peruanos.

(2012). La economía pública en el Perú después del guano y del salitre. Crisis fiscal y élites económicas durante su primer siglo independiente. Lima: Banco Central de Reserva del Perú e Instituto de Estudios Peruanos.

Crozier, R. (1997). El salitre hasta la guerra del Pacífico: una revisión. Historia, vol. 30, pp. 53-126.

Dávalos, P. (1915). Manuel Pardo. Lima: Librería e Imprenta Gil.

Donoso, C. (2003). El puerto de lquique en tiempos de la administración peruana. Historia, vol. 36, pp. 123-158.

(2007). Estado y sociedad en lquique bajo administración peruana 1821-1873 (tesis doctoral). Universidad de Chile, Santiago de Chile.

Echeverría, A. (1934). Vocablos salitreros. Santiago de Chile: Prensas de la Universidad de Chile.

Flores, C. (2000). El civilismo y la cuestión del salitre. Historia. Revista de Historia y Sociedad, nro. 1, pp. 39-47.

Gonzales, O. (2009). Guillermo E. Billinghurst: transfronterizo y multifacético. Socialismo y Participación, nro. 107, pp. 41-52.

González, S. (2000). Guillermo Billinghurst Angulo: una biografía regional. Revista de Ciencias Sociales, nro. 10, pp. 4-22.

(2011). Guillermo Billinghurst, historiador y geógrafo de Tarapacá. En G. Billinghurst, Los capitales salitreros de Tarapacá (pp. ixxlii). Santiago de Chile: Cámara Chilena de la Construcción, Pontificia Universidad Católica de Chile y Biblioteca Nacional.

Hernández, R. (1930). El salitre (Resumen histórico desde su descubrimiento y explotación). Valparaíso: Fisher Hnos.

Kaempffer, E. (1914). La industria del salitre i del yodo. Santiago de Chile: Imprenta Cervantes.

Le, R. y Dagnino, A. (1893). El salitre de Chile o nitrato de soda. Santiago de Chile: Imprenta Cervantes.

Mc Evoy, C. (1997). La utopía republicana. Ideales y realidades en la formación de la cultura política peruana (1871-1919). Lima: Fondo Editorial de la Pontificia Universidad Católica del Perú.

(1994). Un proyecto nacional en el siglo XIX. Manuel Pardo y su visión del Perú. Lima: Fondo Editorial de la Pontificia Universidad Católica del Perú. 
Miller, R. y Greenhill, R. (2011). El Gobierno peruano y el comercio del salitre, 1873-1879. En R. Miller, Empresas británicas, economía y política en el Perú 1850-1934 (pp. 33-67). Lima: Banco Central de Reserva del Perú e Instituto de Estudios Peruanos.

Pinto, J. (1987). Valparaíso: metrópoli financiera del boom del salitre. En S. Lorenzo (Ed.), Valparaíso 1536-1986 (pp. 119-134). Santiago de Chile: Instituto de Historia y Universidad Católica de Valparaíso.

Raimondi, A. (1875). Observaciones al dictamen de los señores Cisneros y García en la cuestión relativa al salitre. Lima: Imprenta de la Opinión Nacional.

Ravest, M. (2008). La casa Gibbs y el monopolio del salitrero peruano: 1876-1878. Historia, vol. I, nro. 41, pp. 63-77.

Rivero, M. (1857). Colección de memorias científicas, agrícolas é industriales publicadas en distintas épocas. Tomo I y II. Bruselas: Imprenta de H. Goemaere.

Salas, M. (2010). El salitre en la vida de los puertos del sur del Perú 18101877: ausencia y riqueza en la aridez del desierto. En Actas del VIII Simposio de Historia marítima y Naval Iberoamericana. 9-13 de noviembre de 2009 (pp. 37-107). Lima: Instituto de Estudios Histórico-Marítimos del Perú.

(2011). La República 1879 a 1883. Historia Marítima del Perú. Tomo XI, vol. 4. Lima: Instituto de Estudios Histórico-Marítimos del Perú.

Semper, E. y Michels, W. (1908). La industria del salitre en Chile. Santiago de Chile: Imprenta, Litografía i Encuadernación Barcelona.

Tantaleán, J. (1983). Política económica-financiera y formación del Estado: siglo XIX. Lima: Centro de Estudios para el Desarrollo y la Participación.

(2012). La gobernabilidad y el leviatán guanero. Desarrollo, crisis y guerra con Chile. Lima: Banco Central de Reserva del Perú e Instituto de Estudios Peruanos. 